\title{
A Multichannel Assignment Scheme for a Wireless Mesh
} Network

\author{
K. Anvesh ${ }^{\# 1}$, CH. Shravan ${ }^{\# 2,}$ N. Anil Babu ${ }^{\# 3}$, G. Mallikarjun Reddy ${ }^{\# 4}$ \\ ${ }^{\# 1}$ Assistant Professor, IT Dept, VCE, Hyderabad, India. \\ ${ }^{\# 2}$ Assistant Professor, CSE Dept.VCE, Hyderabad, India. \\ ${ }^{\# 3}$ Assistant Professor, IT Dept, VCE, Hyderabad, India. \\ ${ }^{\# 4}$ Assistant Professor, CSE Dept.VCE, Hyderabad, India.
}

\begin{abstract}
An emerging technology is wireless mesh network that provides high quality service to end users as the "last mile" of the Internet. A Key technology in wireless mesh networks is Multicast communication. Efficient data distribution among a group of nodes is done by using Multicast. In other types of wireless networks, like sensor networks and MANETs, two multicast schemes are proposed: Level Channel Assignment (LCA) scheme and Dynamic Routing Scheme (DRA) to improve the throughput for multi-channel and multiinterface mesh networks. Multicast trees are built efficiently using thescheme by minimizing the number of relay nodes and total hop count distances of the trees. We use dedicated channel assignment strategies in the schemes to reduce the interference and also to improve the network capacity. Throughput is achieved by using DRA and LCA has a shorter delay be realized in distributed manner.
\end{abstract}

Keywords: Wireless mesh networks, multicast, multi-channel, multi-interface, channel assignment.

\section{Introduction}

A mesh network is created by connecting the individual wireless access point installed at each network user's locally. Provider forwards the data to each network using a next node assignment.An emerging network paradigm is Wireless Mesh Network (WMN) for the next-generation wireless Internet. In these networks we do not have certain constraints that nodes like mobile and do not have power constraints. WMNs are self-organized with the nodes automatically establishing ad hoc networks and maintaining the connectivity[10]. This provides improved reliability as well as larger coverage and reduces equipment cost. Being used for the last mile for extending or enhancing Internet connectivity, commercial deployments of WMNs are already in the works, such as MIT Roofnet [1] and Seattle Wireless [2]. Mesh networks are characterized by the use of multiple channels and multiple interfaces to improve system throughput. In efficient multicast, which cannot be readily achieved through combined unicast or simplified broadcast, is essential to wireless mesh networks and is worthy of thorough investigation. It is often necessary for a portion of end users to retrieve data packets from the Internet.

For example: -A large number of users may watch the FIFA World Cup on the Internet. The gateway that helps to connect the mesh network with the Internet can effectively multicast the data packets to those users. Efficient multicast protocols in WMNs cannot be achieved by adopting or slightly modifying the multicast protocols for other types of multihop wireless networks. Unlike mobile ad hoc networks or wireless sensor networks, route recovery or energy efficiency is not the major concern for mesh networks due to the limited mobility and the rechargeable characteristic of mesh nodes.

\section{Other Related Work}

Moreover, supporting potential major applications, such as Video On Demand, poses a significant challenge for the limited bandwidth of WMNs. Thus, it is necessary to design an effective multicast scheme for mesh networks. Traditional multicast protocols for wireless networks assume that each node is equipped with one interface.

A mesh network provides the nodes with multiple interfaces that can be used to improve the throughput substantially. However, channel assignment is subject to the number of available channels and interfaces, the network topology, the communication requests, and other factors. Interference cannot be completely eliminated due to the limited number of available channels. Level Channel Assignment (LCA) scheme and a Dynamic Routing Scheme to improve throughput for multichannel and multi-interface mesh networks[10].

Simulations show that our schemes greatly outperform the single channel multicast scheme. We observe that MCM achieves better throughput and shorter delay while LCA can be implemented in a distributed manner. 
Desirable qualities of CA schemes

- Minimize connection set-up time

- Adapt to changing load distribution

- Fault tolerance

- Scalability

- Low computation and communication overhead

- Minimize handoffs

- Maximize number of calls that can be accepted concurrently

\section{System Model}

We use some basic terminology and the partial channel conflict phenomena, which is followed by design considerations for multicast schemes in WMNs.

\section{A. Basics}

A mesh network can be designed using a flooding technique or a routing technique. When using a routing technique, the message propagates along a path, by hopping from node to node until the destination is reached. For insuring all its paths' availability, a routing network must allow for continuous connections and reconfiguration around broken or blocked paths, using self-healing schemes. A mesh network whose nodes are all connected to each other is a fully connected network[8]. Mesh networks are composed of three types of nodes: gateways (access points), mesh routers, and mesh clients. Gateways enable the integration of WMNs with various other networks, including the Internet. As dedicated devices provide stable high throughput for mesh clients, mesh routers have minimal mobility and form the mesh backbone. In order to further improve the flexibility and capacity of WMNs, the mesh routers are often equipped with multiple wireless interfaces. As a result, two transmissions of two nearby pairs can be simultaneously scheduled if nonoverlapping channels are assigned [3].

Mesh clients are usually end users, such as laptops and PDAs, which access the Internet through the mesh routers so that the mesh clients are usually within one hop of the mesh routers. Since the multicast packets are always relayed among the mesh backbone, we only consider how to transmit the packets to multiple mesh routers; then packets will be forwarded one more hop to the corresponding mesh clients that desire to receive the packets. Multichannel and multiinterface characteristics enable more concurrent transmissions.

\section{B. Measuring Partial Overlap}

To improve the throughput of WMNs, many studies have been conducted on how to assign orthogonal channels to adjacent wireless links to minimize interference. It is known that $802: 11 \mathrm{~b}=\mathrm{g}$ and $802: 11 \mathrm{a}$ provide 3 and 12 non overlapping channels, respectively. Although 802:11a provides more non overlapping channels than $802: 11 \mathrm{~b}=\mathrm{g}$, it has several drawbacks. Because $802: 11$ a works on a higher frequency spectrum $(5 \mathrm{GHz})$ than $802: 11 \mathrm{~b}=\mathrm{g}(2 \mathrm{GHz})$, it is more difficult to penetrate walls and other obstructions, and thus 802:11a has a shorter range. In addition, the interfaces and access points for 802:11a are more costly. As a result, $802: 11 \mathrm{~b}=\mathrm{g}$ is more commonly used[10]. Previous channel assignment schemes for $802: 11 \mathrm{~b}=\mathrm{g}$ only use three non overlapping channels: 1, 6, and 11. In these studies, a binary interference model is usually assumed, that is, if two links are within interference range of each other, they will interfere with each other if they are on the same channel, and otherwise not. However, the interference can be further reduced by using the partially overlapping channels too, that is, by using any channel from 1 to 11 in the channel assignment.

\section{Level Channel Assignment Scheme}

A common method for multicast is to build a multicast tree, where the source node is usually the gateway. In this paper, we first propose the LCA scheme, which can be achieved by the following steps [10]. First, the nodes obtain their level information. The BFS is used to traverse the whole network. All the nodes are partitioned into different levels according to the hop count distances between the source and the nodes. node a (in level i) and node b (in level i p 1) are within each other's communication range, then a is called the parent of $\mathrm{b}$, and $\mathrm{b}$ is called the child of $\mathrm{a}$. The multicast tree based on the node level information. Initially, the source and all the receivers are included in the tree. 


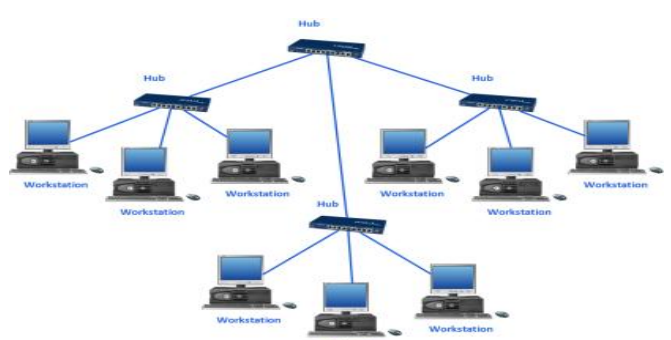

Fig.1 Network topology

Multicast trees-- Multicast routing is about building forwarding trees from the sender $S$ to the group $G$ of receivers or listeners (in IGMP we talk about loyal members). Most efficiently is of course a Shortest Path Tree (SPT). In an SPT each path from the root to all the end nodes is the shortest possible in some sense.

The multicast routing protocol uses either source-based trees or group-shared trees. In some cases, like PIM, first a group-shared tree can be built but routing can build source-based tree later in the process if found more efficient.

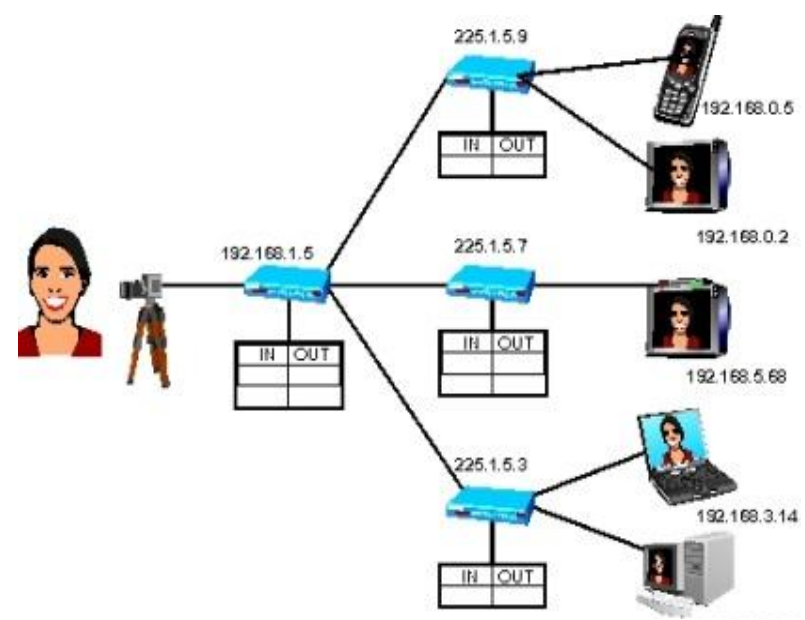

Fig.2 Multicast tree

Then, for each multireceiver $\mathrm{v}$, if one of its parents is a tree node, then connect it with that parent, and stop. This process repeats until all the multireceivers are included in the multicast tree.

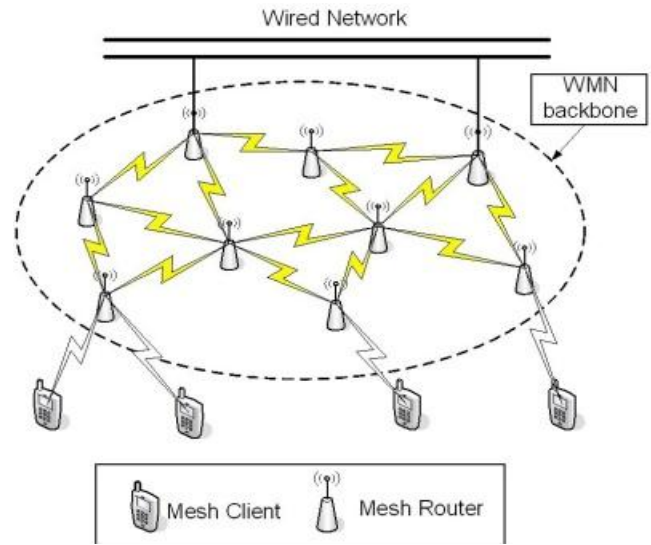

Fig. 3 channel assignment

Reuse distance: minimum distance between two cells using same channel for satisfactory signal to noise ratio.

\section{Go-Ahead Routing System}

Dynamic routing protocols not only perform these path determination and route table update functions but also determine the next-best path if the best path to a destination becomes unusable. The capability to compensate for topology changes is the most important advantage dynamic routing offers over static routing[10]. It dynamically route Packets between each source and its destination so that the chance for system 
break-in,due to successful interception of consecutive packets for a session, is slim. The intention of securityenhanced routing is different from the adopting of multiple paths between a source and a destination to increase the throughput of data transmission.

\section{A. Routing Protocol Basics}

All dynamic routing protocols are built around an scheme. Generally, an scheme is a step-by-step procedure for solving a problem. A routing scheme must, at a minimum, specify the following:

- A procedure for passing reachability information about networks to other routers

- A procedure for receiving reachability information from other routers

- A procedure for determining optimal routes based on the reachability information it has and for recording this information in a route table internetworking

\section{B. Channel Assignment}

The tree node discovery in the previous section allows each multireceiver to connect with the gateway through minimal hop count distance. It show how to assign channels to the interfaces of the tree nodes by proposing two allocation schemes: ascending channel allocation and heuristic channel assignment.

\section{Ascending Channel Allocation}

Each node has two interfaces. Specially, the interface that a node uses to receive packets from its relay node at the upper layer, termed Receive- Interface (RI), is disjoint from the interface the node uses to forward packets to its children, called Send-Interface (SI). In order to guarantee that the relay node can communicate with its children, each node's RI is associated with the SI of its relay node, i.e., they should be assigned the same channel. T

The basic idea of the scheme is straightforward: From top to down in the tree, the channels are assigned to the interfaces in the ascending order until the maximum channel number is reached, then start from channel 0 again. Although simple, this approach avoids the situation that the same channel is assigned to two nearby links that interfere with each other.Although the Ascending Channel Allocation is easy to implement, its performance is still constrained by the limited number of orthogonal channels.Network Throughput can be further improved by exploiting all the partially overlapping channels.

\section{Exploratory Channel Assignment}

'The interference range decreases with the increase of the channel separation. Intuitively, the channel assignment should make a large channel separation for two wireless links if the physical distance between them is short[10]. We aim to minimize the sum of the interference area of all the transmissions. According to the experiment we performed in Section 2, under the condition that all the nodes have the same transmission range $\mathrm{R}$; and $\mathrm{t}$ is the Interference Factor. When allocating a channel for relay node $\mathrm{u}$, the channel assignment should take a channel that minimizes the sum of the square of the IRs between $u$ and us neighboring relay nodes.

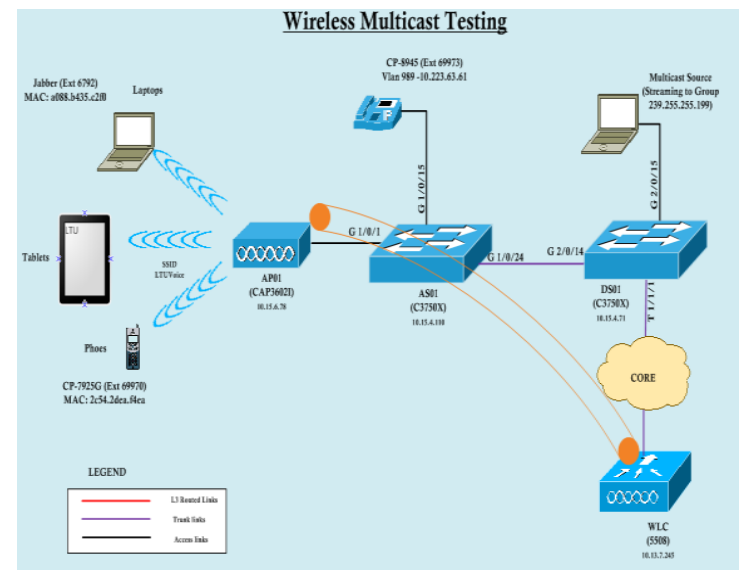

\section{Discussion}

As a basic data communication mechanism, multicast has been intensively studied in Internet and multihop wireless networks. These proposed multicast schemes and protocols do not take advantage of multichannels and multiinterfaces. Multiple gateway test studies that aim to address the channel assignment problem of mesh networks, which is characterized by using multichannels and multi-interfaces. The multicast routing protocols in MANET are classified into three categories according to the way multicast routes are 
created: 1) tree-based, 2) mesh-based, and 3) stateless multicast. In the tree-based protocols, the data packets are transmitted from the source to the destinations along the paths on the multicast tree, which helps to minimize the bandwidth cost [1], [6].

The mesh-based protocols try to create multiple trees among the group members such that the packets can be delivered to each receiver through multiple paths [3], [4], [5]. The alternative paths help to increase the protection against the topology alteration.Static or dynamic methods used to assigning the channels

[4], [8]. They develop a set of centralized or distributed schemes for channel assignments by taking the bandwidth cost, efficient routing, and load balance into account. Most of them believe that static assignment outperforms dynamic assignment due to the channel switching cost and the delay.

New metrics have also been proposed for multihop wireless networks with considering the impact of channel interference, which is used to find high throughput paths between sources and destinations [7], [8]. Our channel assignment is different from previous channel assignment approaches in two aspects: 1) channels based on the multicast structure so that we can exploit the broadcast property of wireless nodes, and 2) full use of the partially overlapping channels instead of just orthogonal channels, which ultimately reduces the interference and improves the throughput.

The tree node discovery in the previous section allows each multireceiver to connect with the gateway through minimal hop count distance. In this section, we discuss how to assign channels to the interfaces of the tree nodes by proposing two allocation schemes: ascending channel allocation and heuristic channel assignment. Here, several channel assignment approaches are compared by QoS.

- Common Channel Assignment

- Load Aware Channel Assignment

- First Random Channel Assignment

FRCA algorithm consists of two basic phases:

1. Initial phase

2. Optimization phase

\section{Motivations}

The direct way to increase the throughput is to reduce the overhead caused by channel assignment. Therefore the primary goal of this design is to use the available frequency bands as efficiently as possible, thus achieve greater throughput. If each node has a dedicated transceiver for each channel, the channel assignment will have zero overhead. This is because every channel is "visible" to every node all the time. However, due to cost consideration, the transceivers are usually fewer than the available channels. Therefore, channel assignment needs to assign the available channel resource to limited transceivers when there are data transmission requests. It is important to know the channel usage information before actual channel assignment. Otherwise, collisions may happen or the extra waiting time will be inevitable.

DCC type schemes collect the channel usage information on commoncontrol channel and assign channels according to it. SP type schemes use time division to clear the past channel usage and use special phase (beginning of each time interval) to do the channel assignment on the default channel.

\section{Conclusion}

Here we discussed about throughput and delay in Level channel assignment scheme in wireless mesh networks where they have the paramount priorities. We proposed a LCA and DRA as two multicast schemes using multichannel and multi-interfaces for improving the efficiency in multicast of WMNs. To minimize the number of the relay nodes and the communication delay an effective multicast structure is constructed.

To reduce the interference the dedicated channel assignment helps as well. Dynamic routing scheme is used for multi path communication, and it also helps in throughput improvement when compared with previous multicast approaches. Our schemes outperform the single-channel multicast in terms of throughput and delay, and more efficient multicast structure and subtle channel assignment can further improve throughput and reduce delay and it is specified by the performance evaluation.

\section{References}

[1]. A. Mishra, V. Shrivastava, and S. Banerjee, "Partially Overlapped Channel Not consider Harmful,"Proc.ACM SIGMETRICS/Performance, 2006

[2]. A.Raniwala and T. cker Chiueh, "Architecture and Schemes or an IEEE 802.11-Based Multi-Channel Wireless Mesh Network,"Proc. IEEE INFOCOM, 2005.

[3]. Chin-Fu Kuo, Member, IEEE, Ai-Chun Pang, Dynamic Routing with Security Considerations, VOL. 20, NO. 1, JANUARY 2009.

[4]. E. Royer and C. Perkins, "Multicast Operation of t he Ad-Hoc on Demand Distance Vector Routing Protocol," Proc. ACM MobiCom, 1999

[5]. J. Tang, G. Xue, and W. Zhang, "Maximum Throughput and Fair Bandwidth Allocation in Multi-Channel Wireless Mesh Networks,'Proc. IEEE INFOCOM, 2006. 
[6]. J. So and N. Vaidya, "Multi-Channel Mac for Ad Hoc Networks: andling Multi-Channel Hidden Terminals Using a Single Transceiver," Proc. ACM MobiHoc, 2004.

[7]. K. Ramachandran, E.M. Belding, K. Almeroth, and M. Buddhiko, "Interference-Aware Channel Assignment in Multi-Radio Wireless Mesh Networks," Proc. IEEE INFOCOM, 2006

[8]. P. Li, N. Scalabrino, Y. Fang, E. Gregori, and I. Chlamtac, “Channel Interference in IEEE 802.11b Systems,” Proc. IEEE Global Telecomm. Conf. (GLOBECOM), 2007.

[9]. Y. Liu and E. Knightly, "Opportunistic Fair Scheduling overMultiple Wireless Channels," Proc. IEEE INFOCOM, 2003.

[10]. K. V. Pradeep, Anvesh K, M.N.P.Swetha Priya, C.Lakshmi,“Level Channel Assignment Scheme For Multichannel Wireless Mesh Network" in IJAST, 2012. 